\title{
EXTENDED FAR-INFRARED CO EMISSION IN THE OMC-1 CORE OF ORION ${ }^{1}$
}

\author{
María J. Sempere, José Cernicharo, Bertrand Lefloch, ${ }^{2}$ and Eduardo González-Alfonso ${ }^{3}$ \\ Consejo Superior de Investigaciones Cientificas, IEM, Departamento de Física Molecular, Serrano 121, \\ Madrid, E-28006, Spain; sempere@astro.iem.csic.es \\ AND \\ SARAH LEEKS \\ Department of Physics, Queen Mary and Westfield College, University of London, \\ Mile End Road, London, E1 4NS, England, UK \\ Received 1999 August 20; accepted 1999 December 17; published 2000 January 31
}

\begin{abstract}
We report on sensitive far-infrared observations of ${ }^{12} \mathrm{CO}$ pure rotational transitions in the OMC-1 core of Orion. The lines were observed with the long-wavelength spectrometer in the grating mode on board the Infrared Space Observatory, covering the 43-197 $\mu \mathrm{m}$ wavelength range. The transitions from $J_{\text {up }}=14$ up to $J_{\text {up }}=19$ have been identified across the whole OMC- 1 core, and lines up to $J_{\text {up }}=43$ have been detected toward the central region, KL/IRc2. In addition, we have taken high-quality spectra in the Fabry-Perot mode of some of the CO lines. In $\mathrm{KL} / \mathrm{IRc} 2$, the lines are satisfactorily accounted for by a three-temperature model describing the plateau and ridge emission. The fluxes detected in the high- $J$ transitions $\left(J_{\text {up }}>34\right)$ reveal the presence of a very hot and dense gas component $\left[T=1500-2500 \mathrm{~K} ; N(\mathrm{CO})=2 \times 10^{17} \mathrm{~cm}^{-2}\right]$, probably originating from some of the embedded sources previously observed in the $\mathrm{H}_{2}$ near-infrared lines. At all other positions in the OMC-1 core, we estimate kinetic temperatures $\geq 80 \mathrm{~K}$ and as high as $150 \mathrm{~K}$ at some positions around IRc2, from a simple large-velocity gradient model.
\end{abstract}

Subject headings: infrared: ISM: lines and bands — ISM: individual (OMC-1) — ISM: molecules line: identification - molecular data — radiative transfer

\section{INTRODUCTION}

The Orion molecular cloud is a prime example of a highmass star-forming region and, due to its proximity, one of the most extensively studied sources. The numerous observations performed in the millimeter and submillimeter range (see Blake et al. 1987 and Genzel \& Stutzki 1989 for a review) have shown that the emission arises from three main components. The bulk of the molecular gas in the extended ridge (with typical densities $\leq 10^{4} \mathrm{~cm}^{-3}$ ) is heated up to $50-60 \mathrm{~K}$ by the UV field of the Trapezium stars. The molecular emission near the high-velocity outflow, the plateau, is distributed in a highly anisotropic region of $40^{\prime \prime}$ in size with a mean density $\leq 10^{6}$ $\mathrm{cm}^{-3}$. The plateau consists of gas heated to temperatures of $80-150 \mathrm{~K}$ and distributed in a low-velocity component that is centered on IRc2 and in a bipolar high-velocity component orthogonal to the latter. Finally, the hot core $(T=400-500 \mathrm{~K})$ of IRc2 is a compact source $\left(10^{\prime \prime}\right)$ with densities up to $10^{7} \mathrm{~cm}^{-3}$. Its high-excitation molecular emission probably arises from material that is shocked by the interaction of the high-velocity outflow with the ambient gas.

The OMC-1 core has been widely studied in various molecular tracers. Among these, $\mathrm{CO}$ is of great interest because observations at different transitions delimit regions with marked contrasts in their physical characteristics. Most are low$J\left(J_{\text {up }} \leq 4\right)$ observations that trace the extended low/moderate density gas and the bulk of the outflow gas. Higher $J$ transitions

\footnotetext{
${ }^{1}$ Based on observations with the Infrared Space Observatory, an ESA project with instruments funded by ESA Member States (especially the PI countries: France, Germany, the Netherlands, and the UK) and with the participation of ISAS and NASA.

${ }^{2}$ Now at L'Observatoire de Grenoble, Grenoble Cédex 9, BP 53, F-38041, France; lefloch@obs.ujf-grenoble.fr.

${ }^{3}$ Also at the Universidad de Alcalá de Henares, Departamento de Física, Campus Universitario, Alcalá de Henares, Madrid, E-28871, Spain.
}

$\left(J_{\text {up }} \geq 6\right)$ are sensitive to the denser and higher temperature gas of the plateau or the hot core; however, they are much less sensitive to those values from the ridge. Multiple CO line observations toward IRc2 carried out with the Kuiper Airborne Observatory (KAO) in the submillimeter/far-infrared range (see, e.g., Boreiko, Betz, \& Zmuidzinas 1989) suggest evidence for hot gas at $600-750 \mathrm{~K}$ in the high-velocity and (possibly) low-velocity components, based on the few flux measurements at $J_{\text {up }}>22$. However, the large uncertainties in the atmospheric transmission yield considerable variations in the parameters of this hot gas component. The Infrared Space Observatory (ISO) satellite offered us the possibility of assessing in a more systematic and comprehensive way the temperature distribution in Orion. We present in this Letter the first large-scale survey of the far-infrared $\mathrm{CO}$ lines in the OMC-1 core of Orion.

\section{OBSERVATIONS AND RESULTS}

Five long-wavelength spectrometer (LWS) grating rasters were performed in the LWS01 mode (in the range of 43-197 $\mu \mathrm{m})$ with a spectral resolution of $0.3-0.6 \mu \mathrm{m}(R=\lambda / \Delta \lambda \sim$ 150-300). A total of 23 positions (six scans per position) with an angular separation of 90" (an approximate beam size) were observed around IRc2. The central position was included in each raster in order to check the relative calibration of the observations. The uncertainties in the absolute calibration are estimated to be $\sim 30 \%$. After prereduction (pipeline 7 ), the data were reprocessed in order to remove nonlinear effects (Leeks et al. 1999). The size of the LWS beam is actually close to $70^{\prime \prime}$ (E. Caux 1999, private communication).

The spectra observed at each position (36 scans for the central one) were averaged together in order to minimize the noise level. The statistical errors of the measured line fluxes are negligible. The transitions at $\lambda<90 \mu \mathrm{m}$ are affected by additional uncertainties in the determination of the continuum 
TABLE 1

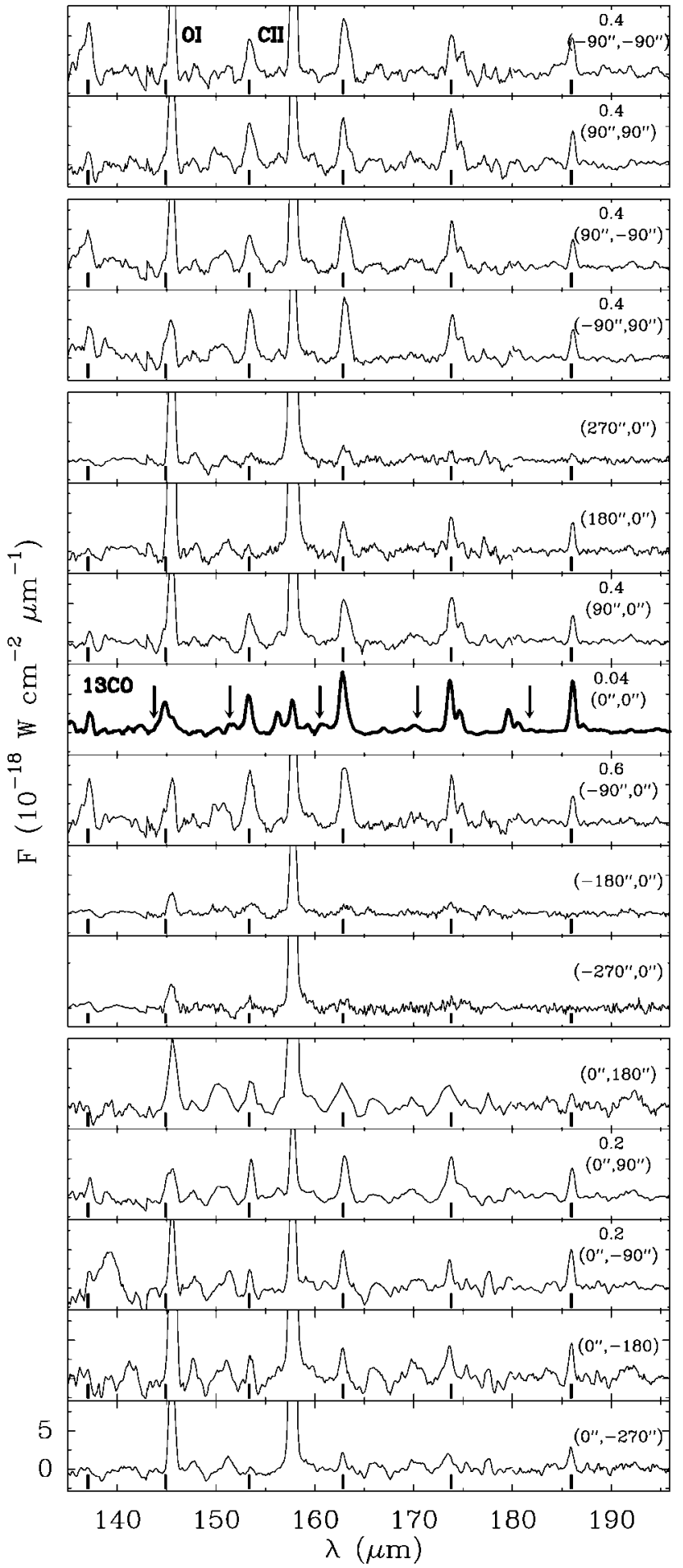

FIG. 1.-LWS grating spectra from the observed positions around IRc2 between 135 and $197 \mu \mathrm{m}$. The lower marks indicate the ${ }^{12} \mathrm{CO}$ rotational transitions from $J_{\text {up }}=14$ to $J_{\text {up }}=19$. At the central position, ${ }^{13} \mathrm{CO}$ transitions are marked by the arrows. Some spectra have been multiplied by a scale factor that is indicated at the top right-hand corner of each panel.

level (hence, the baseline subtraction) as well contamination from neighboring lines. The confusion level is $\sim 3 \times 10^{-18} \mathrm{~W}$ $\mathrm{cm}^{-2}$ in the averaged spectra. However, we checked that all the features identified in the averaged spectra were present in all of the original scans. Figure 1 shows the LWS spectra in
CO Line Fluxes Measured in the Extended Ridge of OMC-1

\begin{tabular}{|c|c|c|c|c|c|}
\hline \multirow[b]{2}{*}{ Position } & \multicolumn{4}{|c|}{ Transition } & \multirow{2}{*}{$\begin{array}{c}T \\
(\mathrm{~K})\end{array}$} \\
\hline & $14-13$ & $15-14$ & $16-15$ & $17-16$ & \\
\hline$\left(0^{\prime \prime},-270^{\prime \prime}\right)$ & 4.9 & 4.6 & 1.7 & 0.22 & \\
\hline$\left(0^{\prime \prime},-180^{\prime \prime}\right)$ & 7.5 & 10 & 6.5 & 2.2 & 80 \\
\hline$\left(0^{\prime \prime},-90^{\prime \prime}\right) \ldots \ldots \ldots \ldots$ & 43 & 42 & 40 & 3.2 & 120 \\
\hline$\left(0^{\prime \prime}, 90^{\prime \prime}\right) \ldots \ldots \ldots \ldots$ & 32 & 48 & 38 & 4 & 120 \\
\hline$\left(0^{\prime \prime}, 180^{\prime \prime}\right) \ldots \ldots \ldots \ldots$ & 2.8 & 4.5 & 2.7 & 1.3 & 80 \\
\hline$\left(-270^{\prime \prime}, 0^{\prime \prime}\right) \ldots \ldots \ldots$ & 0.8 & 2.2 & 0.33 & 0.3 & $\ldots$ \\
\hline$\left(-180^{\prime \prime}, 0^{\prime \prime}\right)$ & 1.9 & 1.3 & 0.13 & & 60 \\
\hline$\left(-90^{\prime \prime}, 0^{\prime \prime}\right)^{*}$ & 16 & 17 & 19 & 1.9 & 120 \\
\hline$\left(90^{\prime \prime}, 0^{\prime \prime}\right)$. & 16 & 18 & 15 & 3.9 & 100 \\
\hline$\left(180^{\prime \prime}, 0^{\prime \prime}\right)$ & 7.1 & 7.0 & 4.5 & 2.3 & 80 \\
\hline$\left(270^{\prime \prime}, 0^{\prime \prime}\right)$ & 2.7 & 3.0 & 0.64 & . & . \\
\hline$\left(-90^{\prime \prime}, 90^{\prime \prime}\right) * \ldots \ldots$ & 16 & 23 & 25 & 6.2 & 100 \\
\hline$\left(90^{\prime \prime},-90^{\prime \prime}\right) \ldots \ldots \ldots$ & 18 & 19 & 25 & 3.8 & 120 \\
\hline$\left(90^{\prime \prime}, 90^{\prime \prime}\right) \ldots \ldots \ldots \ldots$ & 17 & 18 & 6.7 & 2.5 & 80 \\
\hline$\left(-90^{\prime \prime},-90^{\prime \prime}\right) * \ldots \ldots$ & 19 & 29 & 27 & $\ldots$ & 100 \\
\hline
\end{tabular}

Note.-The positions for which the line fluxes $\left(\times 10^{-18} \mathrm{~W}\right.$ $\mathrm{cm}^{-2}$ ) could be contaminated by emission from IRc2 within the LWS error beam are marked with an asterisk (see text).

the range of $135-190 \mu \mathrm{m}$ for the positions where the $\mathrm{CO}$ lines could be unambiguously identified. Besides C II $(158 \mu \mathrm{m})$ and $\mathrm{O}$ I (63 and $146 \mu \mathrm{m}$ ) fine-structure atomic lines, the $\mathrm{CO}$ emission, together with that of $\mathrm{H}_{2} \mathrm{O}$, dominates the OMC- 1 farinfrared spectrum. Among the molecular species detected in Orion at infrared wavelengths, $\mathrm{CO}$ appears to be the most widespread (a full description of the IRc2 spectrum can be found in Cernicharo et al. 1999a and 1999b, hereafter C99a and C99b, respectively). At all of the positions, we observe the pure rotational transitions from $J_{\mathrm{up}}=14$ to $J_{\mathrm{up}}=19$, but only the lines up to $J_{\mathrm{up}}=17$ have a signal-to-noise ratio high enough to allow confident detection outside IRc2. At large angular distances from the central region $\left(>180^{\prime \prime}\right)$, the CO lines become very weak, and the spectra are dominated by the atomic fine-structure lines. The CO-integrated fluxes at 15 selected positions are given in Table 1. Some of the lines are contaminated by nearby features $\left(\mathrm{O}\right.$ I at $145.6 \mu \mathrm{m}, \mathrm{OH}$ at $163.2 \mu \mathrm{m}$, and $\mathrm{H}_{2} \mathrm{O}$ at different wavelengths), whose contributions have been estimated from complementary Fabry-Perot (FP) observations (C99a) or from the grating data in which the line separation was about $0.3 \mu \mathrm{m}$.

Toward the central region, the consecutive pure rotational lines from $J_{\text {up }}=14$ up to $J_{\text {up }}=43$ could be identified. Due to the presence of numerous unresolved components, the calibration of the high- $J$ transitions $\left(J_{\text {up }} \geq 34\right)$ is somewhat uncertain. When observing the $\mathrm{H}_{2} \mathrm{O}$ lines toward IRc2 at high spectral resolution with the LWS in the FP mode $(R \sim 7000)$, several CO transitions, between $J_{\text {up }}=14$ and $J_{\text {up }}=33$, were also detected in some detector bands (Fig. 2; for details on the observations, see C99a). The fluxes agree to better than $30 \%$ with the grating data, except for the $J_{\text {up }}=16$ and the $J_{\text {up }}=28$ lines where the differences reach $50 \%$. In view of the possible uncertainties in the actual pipeline, we have used the grating fluxes for these two lines. Quite remarkably, the line intensities are nearly constant from $J_{\text {up }}=18$ up to $J_{\text {up }}=21$, indicating that the lines are optically thick and that the emitting regions are similar in size. The line intensity merely decreases by a factor of 3 for the following transitions up to $J_{\text {up }}=28$. All of the lines have typical widths of $\Delta v \simeq 60 \mathrm{~km} \mathrm{~s}^{-1}$ (half-power full width); i.e., they are partially resolved by the LWS in the FP mode. 


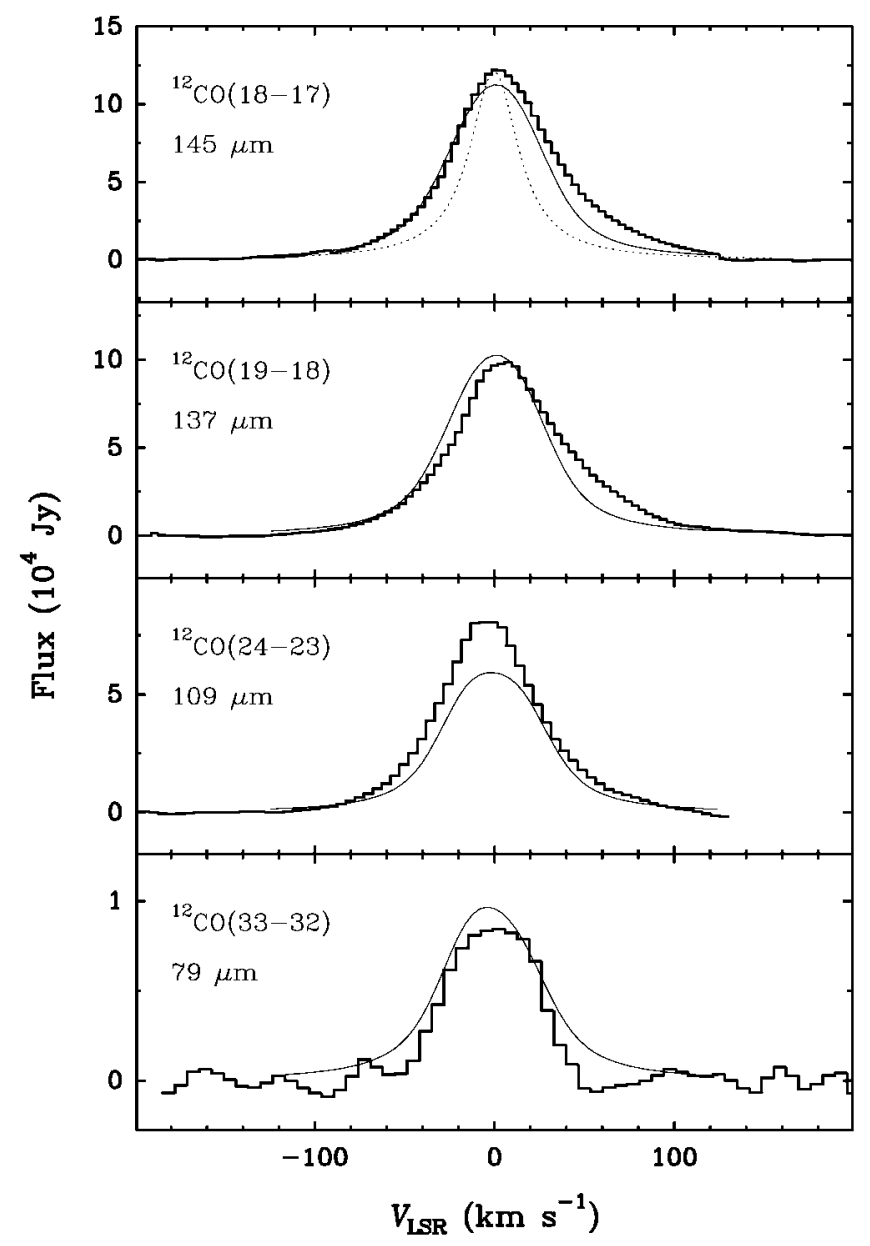

FIG. 2.-A montage of select $\mathrm{CO}$ transitions observed toward IRc2 with the LWS in the FP mode (thick line). We have indicated (thin line) the modeled emission of the plateau (see text). The instrumental response of the FP at $145 \mu \mathrm{m}$ is indicated on the $J_{\text {up }}=18$ spectra (dotted line).

\section{DISCUSSION}

\subsection{The Ridge}

In order to determine the temperature of the gas that was traced by the CO lines detected in the OMC-1 core, we have modeled the emission of a gas layer with a column density of $N(\mathrm{CO})=10^{19} \mathrm{~cm}^{-2}$ for various densities, $n\left(\mathrm{H}_{2}\right)$, and temperatures. The $\mathrm{CO}$ fluxes were computed using a simple largevelocity gradient (LVG) approach. For the LWS range, the dust opacity is still low enough that the coupling between dust and gas can be neglected. The adopted line width in the model is $\Delta v=10 \mathrm{~km} \mathrm{~s}^{-1}$ for positions close to the center $\left( \pm 90^{\prime \prime}, \pm 90^{\prime \prime}\right)$ and $\Delta v=5 \mathrm{~km} \mathrm{~s}^{-1}$ for the points more distant from IRc2.

We display in Figures $3 b-3 d$ the expected fluxes for the transitions $J_{\text {up }} \leq 25$ with $n\left(\mathrm{H}_{2}\right)$ densities in the range (4-40) $\times 10^{4} \mathrm{~cm}^{-3}$ for specific temperatures. The temperatures shown are those that give the best match to the fluxes measured at three typical positions in the core: halfway between the bar and the main core $\left(90^{\prime \prime},-90^{\prime \prime}\right)$, south of IRc2 toward the S6 protostellar source $\left(0^{\prime \prime},-90^{\prime \prime}\right)$, and the extended ridge $\left(0^{\prime \prime},-180^{\prime \prime}\right)$. For densities in the range $(4-40) \times 10^{4} \mathrm{~cm}^{-3}$, which is typical of those measured in the OMC-1, temperatures larger than $80 \mathrm{~K}$ are required to account for all of the observed fluxes. Lower temperatures of $\sim 60 \mathrm{~K}$ fail to reproduce the
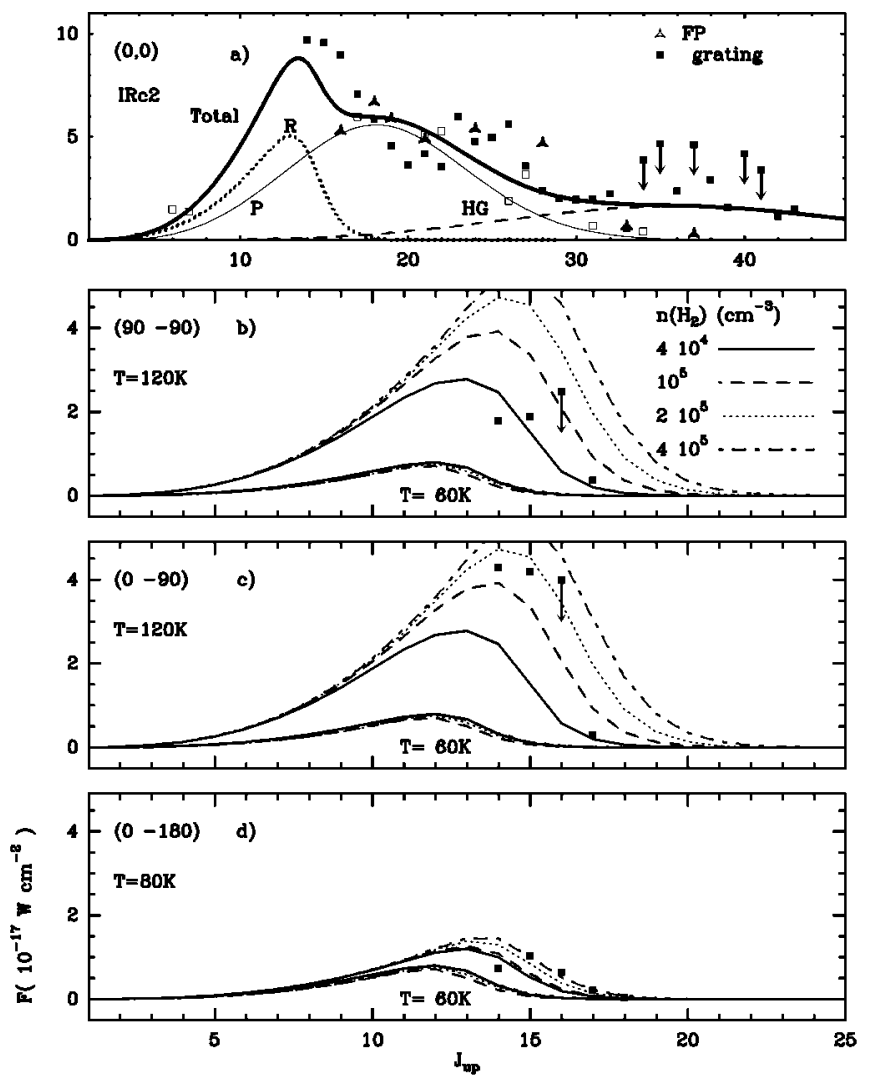

FIG. 3.-(a) Integrated flux of the $\mathrm{CO}$ lines detected toward the IRc2 region. The modeled contributions from the plateau $(P$, thin solid line $)$ and the ridge $(R$, thin dotted line $)$ are shown. A hot gas component (HG) was introduced (dashed line) in order to reproduce the emission at $J_{\text {up }}>34$. The arrows mark the fluxes of the observed $\mathrm{CO}$ lines that are possibly overestimated because of contamination by weak adjacent lines. We have superposed (open squares) the values estimated for the $I S O$ beam size from previous work: $J_{\text {up }}=4$ (Schultz, Krügel, \& Beckmann 1992), 6 (Graf et al. 1990), 7 (Schmid-Burgk et al. 1989; Howe et al. 1993), 21 (Boreiko \& Betz 1989), and 22-34 (Watson et al 1985). ( $b-d$ ) Expected fluxes for the CO lines up to $J_{\text {up }}=25$ observed in the extended ridge with the ISO LWS beam, for kinetic temperatures comprised of 60-120 K, $\mathrm{H}_{2}$ densities (4-40) $\times 10^{4} \mathrm{~cm}^{-3}$ and a column density $N(\mathrm{CO})=10^{19} \mathrm{~cm}^{-2}$. We show the "best-temperature" fit for each pointing, which accounts for the measured fluxes (Table 1). The arrows have the same meaning as above.

observations by a factor of 2 or more (see Fig. 3). Around the central position $\left( \pm 90^{\prime \prime}, \pm 90^{\prime \prime}\right)$, we find evidence for warm gas with $T \geq 120 \mathrm{~K}$ and densities on the order of $\sim 10^{5} \mathrm{~cm}^{-3}$. The column density adopted in our model appears as an upper limit since it was observed toward the IRc2 core (Blake et al. 1987). If we adopt a lower column density of $5 \times 10^{18} \mathrm{~cm}^{-2}$ for positions that are remote from IRc2, in closer agreement with CO observations at millimeter wavelengths (Bally et al. 1987), reasonable fits are obtained by increasing the kinetic temperature by $10 \%-20 \%$ or, conversely, by increasing the density by a factor of 2-3. This does not effect our conclusions about the extended warm component around the hot core. From Figure 3, it appears that low- $J$ observations bring very little in the way of constraints to the temperature determinations in our modeling. The kinetic temperatures derived from KAO observations of the $J_{\text {up }}=7$ line $(\sim 90-100 \mathrm{~K})$ at 100 " resolution by Schmid-Burgk et al. (1989) agree reasonably well for the S6 region and close to IRc2.

On the western side of the ridge, our modeling requires 
temperatures above $100 \mathrm{~K}$ or more and/or high column densities and volume densities to account for the observed fluxes (see Table 1). The KAO data suggest moderate temperatures around $\sim 50 \mathrm{~K}$, although it is difficult to compare both sets of data because of the larger beam and coarse sampling of the former. We note that some contamination in our data from the IRc2 region within a possible LWS error beam cannot be excluded and could reduce the derived column densities and/or kinetic temperatures. Also, the LWS beam profile is known to be rotationally asymmetric for an extended source and could account for some of the discrepancies.

\subsection{The Orion-KL/IRc2 Region}

The emission in the far-infrared $\mathrm{CO}$ lines from $J_{\text {up }}=18$ to 33 can be reasonably explained by a simple two-temperature model of the plateau region. First we note that a single-temperature model cannot reproduce the flux of both the high$\left(J_{\text {up }}>28\right)$ and low- $J$ lines. On the other hand, the range of temperatures reported in the extended ridge imply that its contribution becomes negligible with respect to the plateau (see also Fig. 3 and below) for transitions above $J_{\text {up }}=18$. The physical properties of the gas in the different regions (in particular, the plateau) were chosen as close as possible to those of Blake et al. (1987). The low-velocity plateau is modeled as a twoshell region expanding at $25 \mathrm{~km} \mathrm{~s}^{-1}$ with a microturbulent velocity of $10 \mathrm{~km} \mathrm{~s}^{-1}$. The line fluxes were calculated with the radiative transfer code developed by González-Alfonso \& Cernicharo (1997) and were convolved with the expected instrumental profile (see Fig. 2), which accounts for the FP instrumental response (E. Caux 1999, private communication). We have adopted standard properties for the dust (silicate) grains: a typical radius of $0.1 \mu \mathrm{m}$ and a standard gas-to-dust mass ratio of 100. The inner region gives rise to the entire emission of the $J_{\text {up }}=33$ and $J_{\text {up }}=28$ lines, to the bulk of the emission of the $J_{\text {up }}=24$ line and to a significant contribution of the lower $J$ lines. The colder gas of the plateau also contributes to the emission of the low- $J$ lines. Based on our study of the $\mathrm{H}_{2} \mathrm{O}$ emission in Orion by C99a, we assume that the density is high enough to almost thermalize the CO lines: $\sim 10^{7} \mathrm{~cm}^{-3}$ for the inner region and $\sim 10^{6} \mathrm{~cm}^{-3}$ for the external one, which are close to the estimates derived by Blake et al. (1987). We use the standard $\mathrm{CO}$ abundance of $1.2 \times 10^{-4}$. The ratio of the $J_{\text {up }}=33$ to the $J_{\text {up }}=28$ lines sets an upper limit of $\sim 500 \mathrm{~K}$ to the temperature of the inner region: a higher value would imply a lower contrast between the two lines for any CO column density. For a lower temperature $(\sim 350 \mathrm{~K})$, the high column density needed to account for the $J_{\text {up }}=33$ line would then overestimate the ${ }^{13} \mathrm{CO}$ lines, whereas they are hardly detected (we adopt an upper limit of $3000 \mathrm{Jy}$ ). We have adopted a temperature $T=400 \mathrm{~K}$ for the inner plateau region. The resulting column density is $N(\mathrm{CO})=10^{19} \mathrm{~cm}^{-2}$; the outer and inner radii are $3 \times 10^{16}\left(4^{\prime \prime} .5\right)$ and $2.2 \times 10^{16} \mathrm{~cm}$, respectively. For the external shell (outer radius $=7 \times 10^{16} \mathrm{~cm}$ ), the temperature is $T=300 \mathrm{~K}$, and the column density $N(\mathrm{CO})=$ $3.5 \times 10^{18} \mathrm{~cm}^{-2}$. The assumed column densities agree with the values quoted in the literature (Blake et al. 1987).

The agreement with the line profiles is very satisfying when taking into account the simple hypothesis used. The apparent high-velocity emission is fully reproduced by convolving it with the broad-wing instrumental response (Fig. 2). Down to the sensitivity of these observations, we do not detect in this wavelength range any emission from the very high velocity gas around IRc2. Comparison with all the lines observed by the LWS (in either FP or grating mode) proves to be satisfying up to the $J_{\text {up }}=33$ transition. We have added to our modeling the contribution of the extended ridge, as a layer at $T=80 \mathrm{~K}$ (see $\S 3.3$ ) with a column density of $N(\mathrm{CO})=4 \times 10^{19} \mathrm{~cm}^{-2}$. The resulting fit and the contributions of the different regions are shown in Figure 3. As a whole, the emission observed toward the IRc2 core can be satisfactorily accounted for by two regions of the plateau gas at different temperatures, $~ 300$ and $\sim 400 \mathrm{~K}$, and from the ridge contributing significantly up to the $J_{\text {up }}=16$ line. Note that since the central part of the line profiles is only partially resolved by the FP mode, we cannot exclude that a part of the modeled warm component actually arises from the hot core (where $\Delta v \approx 5 \mathrm{~km} \mathrm{~s}^{-1}$ ). Therefore, the emission from the $T=400 \mathrm{~K}$ component should be considered as the combined emission of the plateau and the hot core. The contribution of the ridge seems to be underestimated in our model. However, as for the adjacent positions, the contribution from an error beam could effect the observed fluxes.

\subsection{The High-J CO Lines}

The higher $J$ transitions $\left(J_{\text {up }}>34\right)$ observed with the grating mode are largely contaminated by other emission lines. Using our FP data, we have estimated the contribution of the strongest adjacent lines $\left(\mathrm{OH}, \mathrm{H}_{2} \mathrm{O}\right.$, and $\left.\mathrm{NH}_{3}\right)$ to the $\mathrm{CO}$ fluxes. In view of the high density of spectral lines for $\lambda<90 \mu \mathrm{m}$, additional contamination by other weaker lines cannot be discarded (C99a; C99b). We note, however, that the lines are well above the confusion level and that most of the observed flux must arise from $\mathrm{CO}$ itself.

After correction, all the CO lines observed in either FP or grating mode lie above the model of the plateau and the ridge (Fig. 3a), showing evidence of a hotter gas component. In the absence of other information on the spatial location and the kinematics of this gas, we speculate that such emission arises from the shocked regions of the interaction of the Orion outflows with the ambient medium, detected in the $2.12 \mu \mathrm{m}$ lines. All sources of strong $\mathrm{H}_{2}$ emission (BN, KL, PK1, and PK2; Beckwith et al. 1983) are located within our 70" beam. We modeled the high- $J$ CO emission using an LVG approach with parameters representative of the $\mathrm{H}_{2}$ peak 1 region: a size of $10^{\prime \prime}$, a density $n\left(\mathrm{H}_{2}\right) \sim 3 \times 10^{7} \mathrm{~cm}^{-3}$ for the shocked emitting region, and a line width $\Delta v=30 \mathrm{~km} \mathrm{~s}^{-1}$. The fluxes are roughly accounted for by a gas layer at $T \sim 1500-2000 \mathrm{~K}$ and a column density $N(\mathrm{CO}) \sim 6 \times 10^{17} \mathrm{~cm}^{-2}$, implying a shell thickness of $1.6 \times 10^{14} \mathrm{~cm}$. These values are merely indicative, because of the lack of spectral and spatial information, but are consistent with those predicted by shock models (e.g., Flower \& Pineau des Forêts 1999).

This work has been partially supported by the Spanish DGES under grant PB96-0883 and by PNIE grant ESP97-1618-E. S. L. acknowledges receipt of a PPARC award. We thank S. Pérez-Martínez and J. R. Goicoechea for their help in the data reduction and E. Caux for providing us with the Fabry-Perot instrumental profile. 


\section{REFERENCES}

Bally, J., Stark, A. A., Wilson, R. W., \& Langer, W. D. 1987, ApJ, 312, L45 Beckwith, S., Evans, N. J., II, Gatley, I., Gull, G., \& Rusell, R. W. 1983, ApJ, 264, 152

Blake, G. A., Sutton, E. C., Masson, C. R., \& Phillips, T. G. 1987, ApJ, 315, 621

Boreiko, R. T., \& Betz, A. L. 1989, ApJ, 346, L97

Boreiko, R. T., Betz, A. L., \& Zmuidzinas, J. 1989, ApJ, 337, 332

Cernicharo, J., et al. 1999a, in The Universe as Seen by ISO, ed. P. Cox \& M. F. Kessler (ESA SP-427; Noordwijk: ESA/ESTEC), 565 (C99a) 1999b, in The Universe as Seen by ISO, ed. P. Cox \& M. F. Kessler (ESA SP-427; Noordwijk: ESA/ESTEC), 651 (C99b)

Flower, D. R., \& Pineau des Forêts, G. 1999, MNRAS, 308, 271

Genzel, R., \& Stutzki, J. 1989, ARA\&A, 27, 41
González-Alfonso, E., \& Cernicharo, J. 1997, A\&A, 322, 938

Graf, U. U., Genzel, R., Harris, A. I., Hills, R. E., Rusell, A. P. G., \& Stutzki, J. 1990, ApJ, 358, L49

Howe, J. E., Jaffe, D. T., Grossman, E. N., Wall, W. F., Mangum, J. G., \& Stacey, G. J. 1993, ApJ, 410, 179

Leeks, S. J., Swinyard, B. M., Lim, T. L., \& Clegg, P. E. 1999, in The Universe as Seen by ISO, ed. P. Cox \& M. F. Kessler (ESA SP-427; Noordwijk: ESA/ESTEC), 81

Schmid-Burgk, J., et al. 1989, A\&A, 215, 150

Schultz, A. J., Krügel, E., \& Beckmann, U. 1992, A\&A, 264, 629

Watson, D. M., Genzel, R., Townes, C. H., \& Storey, J. W. V. 1985, ApJ, 298, 316 